\title{
Emerging complication of laparoscopic power morcellation: A rare case of parasitic leiomyoma
}

\author{
W A B Weerakoon ${ }^{a}$, S Mahesan ${ }^{b}$, S K Chandrasinghe ${ }^{c}$, S N Samarakkody ${ }^{d}$, M A M M Jayawardena ${ }^{e}$
}

\begin{abstract}
Introduction

A parasitic leiomyoma is considered an extremely rare variant of extra uterine leiomyoma. It presents as a peritoneal pelvic benign smooth muscle mass separate from the uterus.

We report a case of a 36-year-old female who presented with generalized abdominal pain and was
\end{abstract}

found to have a parasitic fibroid which may have occurred following laparoscopic myomectomy, and along with it a review on current literature of parasitic fibroids.

Parasitic leiomyoma presents with vague symptoms and the diagnosis is challenging. In prevention of this condition, various alternatives must be utilized when using electromechanical power morcellation for myomectomy.

Key words: Parasitic leiomyoma, laparoscopic myomectomy, electro mechanical morcellation

Sri Lanka Journal of Obstetrics and Gynaecology

2019; 41: 20-23

DOI: http://doi.org/10.4038/sljog.v41i1.7878

${ }^{a}$ Registrar in Obstetrics and Gynaecology, Department of Obstetrics and Gynaecology, Colombo South Teaching Hospital, Kalubowila, Sri Lanka

${ }^{\mathrm{b}}$ Registrar in Obstetrics and Gynaecology, Department of Obstetrics and Gynaecology, Colombo South Teaching Hospital, Kalubowila, Sri Lanka

' Registrar in Obstetrics and Gynaecology, Department of Obstetrics and Gynaecology, Colombo South Teaching Hospital, Kalubowila, Sri Lanka

'Senior Registrar in Obstetrics and Gynaecology, Department of Obstetrics and Gynaecology, Colombo South Teaching Hospital, Kalubowila, Sri Lanka

e Senior Lecturer in Obstetrics and Gynaecology, Department of Obstetrics and Gynaecology, Faculty of Medical Sciences, University of Sri Jayewardenepura, Sri Lanka

Correspondence: WABW, e-mail: <buddhikaweerakoon@gmail.com>

Received 12 $2^{\text {th }}$ December 2018 and revised version accepted $2^{\text {nd }}$ March 2019.

http://orcid.org/0000-0002-5863-3324

Competing interest: The authors report no conflict of interest

This is an open-access article distributed under the terms of the Creative Commons Attribution 4.0 International License, which permits unrestricted use, distribution and reproduction in any medium provided the original author and source are credited. 


\section{Introduction}

Leiomyomas are the most commonly occurring benign tumors of the uterus which are monoclonal in origin. Prevalence is about 25\% in all females of reproductive age group based on clinical presentation ${ }^{1}$. Parasitic leiomyoma is considered an extremely rare variant of extra uterine leiomyoma. It presents as a benign smooth muscle mass separate from the uterus ${ }^{2}$.

When a subserosal leiomyoma adheres to surrounding structures such as omentum it acquires its blood supply from the omental vessels, detaches from the uterine pedicle and becomes an extrauterine fibroid.

With popularity of minimal invasive surgical technology most of the myomas are excised laparoscopically and specimens are retrieved by power morcellation. Fragmentation of the fibroids during morcellation may lead to peritoneal scattering, and if not identified at the time of the surgery, may grow into parasitic leiomyomas ${ }^{3}$.

When leiomyomas occur with unusual growth patterns or in unusual sites, it makes their identification difficult clinically and radiologically. Also, the management becomes challenging. Therefore, we report a case of a 36-year-old female with a parasitic fibroid following laparoscopic myomectomy, and a review on current literature of parasitic fibroids.

\section{Case report}

A 36-year-old unmarried lady presented with on and off generalized mild abdominal pain. She had undergone laparoscopic myomectomy one year back for fibroid uterus with heavy menstrual bleeding not responding to medical therapy. The previous operative findings had revealed an enlarged uterus with multiple fibroids. The largest fibroid was intramural, measuring $10 \times 10 \mathrm{~cm}$ in size in the fundal region. Two other fibroids, one intramural $6 \times 4 \mathrm{~cm}$ sized over the anterior wall and the other sub-serosal $4 \times 4 \mathrm{~cm}$ sized over the posterior wall were noted. Specimens had been retrieved via power morcellation according to the previous surgical notes.

On the index admission she was investigated for generalized abdominal pain and chronic pelvic pain. On examination she was found to have a soft abdomen with no palpable masses. Abdominal ultrasound showed a normal sized uterus with a $2.5 \times 2 \mathrm{~cm}$ anterior lower uterine fibroid and a small posterior wall fibroid. Abdominal computed tomography scan showed a $5 \times 5 \mathrm{~cm}$ mass at the right hypochondrial region, possibly a dermoid tumour of small bowel mesentery or a gastrointestinal stromal tumour arising from upper small bowel. As there was a diagnostic dilemma, diagnostic laparoscopy was performed, and the mass was found to be a parasitic fibroid, $5 \times 5 \mathrm{~cm}$ in size at the right hypochondrial region just underneath the liver. Blood supply to the fibroid was derived from the anterior abdominal wall and the omentum. The peritoneal cavity was thoroughly explored for presence of any other masses, and none were found. The fibroid was released from the surrounding adjacent organs, retrieved via the endobag and sent for histopathology. Histopathology confirmed the leiomyoma.
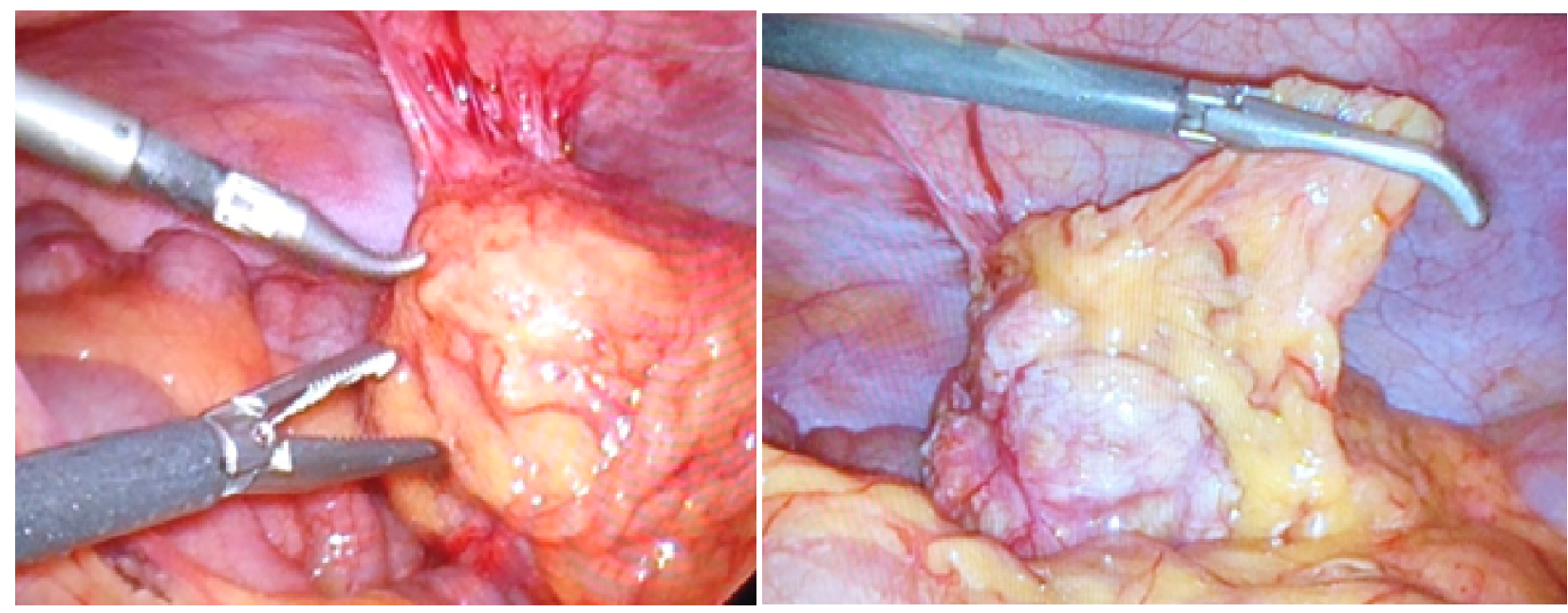


\section{Discussion}

The laparoscopic approach towards myomectomy has gained increasing popularity due to the reduced postoperative pain and discomfort, shorter recovery time and hospital stay, and fewer complications. Electromechanical power morcellator is used to divide large leiomyomas into small fragments and remove them from the peritoneal cavity. During this process some of the resultant small fragments may unintentionally remain in the peritoneal cavity and become implanted, leading to subsequent growth ${ }^{4}$. These parasitic leiomyomas become a rare late sequela of laparoscopic myomectomy and has an incidence of $0.12-0.95 \%$. Median diagnosis interval is 48 months ${ }^{5}$.

After the introduction of the electromechanical morcellator, the first parasitic myoma was reported in 1997 by Ostrzenski following laparoscopic morcellation ${ }^{6}$. Most of the reported cases in last few decades are secondary to laparoscopic procedures involving the uterus.

It has a quite nonspecific clinical presentation depending on the site of recurrence. Commonest sites include the pelvic cavity/wall, rectum, vagina or cervical stump, caecum, small intestine and laparoscopic port site ${ }^{7}$. In our patient it was found at the right hypochondrial region just underneath the liver.

Most patients are asymptomatic and incidental discovery of the parasitic myoma occurs during investigations for other conditions, especially if they are smaller in size. Yet when symptomatic they usually present with abdominopelvic mass, vague abdominal pain or pressure, abdominal distention, or pervaginal bleeding. As these fibroids grow in ectopic sites they may compress surrounding organs and peritoneum, causing nonspecific pain symptoms.

When considering differential diagnoses in a patient who presents with an intraabdominal mass, it is important to take into account her past surgical history. We had not thought of the diagnosis of parasitic fibroid preoperatively, although she had a history of past laparoscopic myomectomy.

Definitive diagnosis requires histological confirmation, but no specific diagnostics are available due to its nonspecificity. Measurement of tumor markers such as CA 19.9, CA 125 and alphafeto protein can be offered but these too are not specific ${ }^{8}$.
Treatment involves laparoscopic excision of the parasitic myoma, which gives good outcomes compared to laparotomy if malignancy is not suspected ${ }^{8}$. In our patient we had a diagnostic dilemma. Therefore, we planned for a diagnostic laparoscopy, and excision was performed laparoscopically. A complete peritoneal survey was done to locate any other missed masses.

Various techniques have been suggested to reduce the incidence of parasitic fibroids. Power morcellation in to a containment bag (Endo Bag) reduces spread of the morcellated fragments ${ }^{7}$. However, morcellation should be avoided in the case of a suspected malignancy. Large specimens can be bisected or cut in to small pieces and retrieved via colpotomy or mini laparotomy. Comprehensive inspection of the whole peritoneal cavity is essential after power morcellation to avoid leaving behind any fragments.

In a patient with a nonspecific intra-abdominal mass with a history of previous laparoscopic myomectomy it is essential to consider parasitic fibroid as a differential diagnosis although it is a rare condition.

\section{Conclusion}

Parasitic leiomyoma is an extremely rare subtype of uterine leiomyoma. It presents with vague symptoms and the diagnosis is challenging. The management is usually careful resection from the surrounding structures and removal of the leiomyoma.

\section{References}

1. Salih AM, Kakamad FH, A. H. D, J. Habibullah I, M. Rauf G. Parasitic leiomyoma: A case report with literature review. Int J Surg Case Rep [Internet]. 2017;41:33-5. Available from: http:// dx.doi.org/10.1016/j.ijscr.2017.10.003

2. Dashraath P, Lim LM, Huang Z, Ilancheran A. Parasitic leiomyoma. Am J Obstet Gynecol [Internet]. 2016;215(5):665.e1-665.e2. Available from: http://dx.doi.org/10.1016/j.ajog.2016.07.017

3. Nezhat $\mathrm{C}$, Kho K. Iatrogenic myomas: new class of myomas? J Minim Invasive Gynecol. 2010; 17(5): 544-50.

4. Tulandi T, Leung A, Jan N. Nonmalignant Sequelae of Unconfined Morcellation at Laparoscopic Hysterectomy or Myomectomy. J Minim Invasive Gynecol. 2016; 23(3): 331-7.

5. Van Der Meulen JF, Pijnenborg JMA, Boomsma CM, Verberg MFG, Geomini PMAJ, Bongers MY. 
Parasitic myoma after laparoscopic morcellation: A systematic review of the literature. BJOG An Int J Obstet Gynaecol. 2016; 123(1): 69-75.

6. Ostrzenski A. Uterine leiomyoma particle growing in an abdominal-wall incision after laparoscopic retrieval. Obstet Gynecol [Internet]. 1997;89(5 II SUPPL.):853-4. Available from: http://dx.doi.org/ 10.1016/S0029-7844(97)81428-7
7. Oindi FM, Mutiso SK, Obura T. Port site parasitic leiomyoma after laparoscopic myomectomy: a case report and review of the literature. 2018; 10-3.

8. Lete I, Gonzalez J, Ugarte L, Barbadillo N, Lapuente O, Alvarez-Sala J. Parasitic leiomyomas: a systematic review. Eur J Obstet Gynecol Reprod Biol. 2016 Aug; 203: 250-9. 